Received 26 ${ }^{\text {th }}$ August 2020, Accepted $24^{\text {th }}$ September 2020

Link to DOI:

10.25220/WNJ.V04.S2.0006

Journal Website:

www.worldnutrijournal.org

\section{Benefit of synbiotic intervention in Caesarean section born infants and children: A nutritional perspective}

\author{
Saptawati Bardosono ${ }^{1}$, Dian Novita Chandra ${ }^{1}$ \\ 1. Department of Nutrition, Medical Faculty, Universitas Indonesia-Cipto Mangunkusumo National \\ General Hospital, Jakarta, Indonesia
}

\begin{abstract}
The benefit to have infants being delivered physiologically through vagina is amongst others to get maternal microbes transmission that will affect host immunity and metabolic development. However this mode of delivery is not always the choice to infants for several reasons. Therefore, it raises questions whether there is a need to give specific intervention to the caesarean section (Csection) born infants and children for their optimal growth and development, i.e. provision of nutrients with or without pre-, pro- or synbiotics. Nutritional intervention is certainly important to support growth and development of all children, especially those born by C-section. However, in addition, to anticipate perturbation in the gut microbiota there is a need to prepare the C-section born infant through translocation from the mother's intestinal microbiota, early initiation of breastfeeding and/or synbiotic supplementation formula. The superiority of synbiotic compare to prebiotic or probiotic alone is that synbiotic thought to have synergistic beneficial effects on the immune and metabolic systems in which it can compensates the delayed Bifidobacterium colonization modulates the production of acetate and the acidification of the gut in C-section born infants. However, we still need to find consistent evidence \& recommendation in the world on synbiotic for children in general and specifically for the C-section born infant \& children.
\end{abstract}

Keywords: Caesarean section, children, gut microbiota, synbiotic

\section{Introduction}

Pregnancy is triggering maternal inflammation due to several physiological changes which results in prenatal stress. In addition, the absence of vaginal microbiota caused by Caesarean section (C-section) delivery mode will alter infant's gut microbiota, or so called dysbiosis. There are several theoretical outcomes of dysbiosis, in which through the increase

\footnotetext{
Corresponding author:

Dian Novita Chandra

Department of Nutrition, Medical Faculty

Universitas Indonesia - Cipto Mangunkusumo

National General Hospital, Jakarta, Indonesia

Email:diannovitach@yahoo.com
}

of blood brain barrier will result to neuroinflammation and will cause abnormal neuronal/brain development. On the other hand, dysbiosis will result to gastro-intestinal dysfuntion or leaky gut that will impair the parasympathetic system function (i.e. vagus nerve) and will cause cognitive and behavioral deficits. While through metabolic disorder caused by dysbiosis, there will be disturbed cross-talk innate immune system and dysregulation of systemic (adaptive) immune system that will result to allergy and autoimmunity. ${ }^{1}$

How could mode of delivery increase the risk of poor child growth and development? Although there is limited evidence on the association between $\mathrm{C}$ section birth and linear growth and/or brain development, a retrospective cohort study among mothers having children aged 6-24 months old in 
Ghana found that compared to the C-section infants, vaginally delivered infants were 1.8 times more likely to receive adequate neonatal feeding, and after controlling for potential confounding factors, there's a significant increment by 0.121 standard units on linear growth as measured by height-for-age (HAZ). ${ }^{2}$ On the other hand, a cross-sectional study done in East China found that $67.3 \%$ were born by C-section, in which $15.7 \%$ of those preschool children aged 3-6 years old were obese. And, after adjusted for parental factors, child characteristics and family income, the odd of overweight and obesity was 1.35 and 1.25 respectively. ${ }^{3}$

Furthermore, in relation to brain development, a retrospective study in Arkansas Children's Hospital and Brown University USA found that by using diffusion tensor imaging, myelin water fraction imaging, voxel-based morphometry, and/or restingstate functional magnetic resonance imaging (fMRI), C-section delivery may influence infant brain development. ${ }^{4}$ To evaluate the relation between $\mathrm{C}$-section birth and child cognitive development, a longitudinal study of Australian children aged 4 to 9 years revealed that C-sectionborn children perform significantly below by up to a tenth of a standard deviation in national numeracy test score at age 8-9 years compared to vaginallyborn children ${ }^{5}$ To explore the mechanism on how Csection delivery could affect child growth and development in relation to gut microbiota, there is an impact of external factors on the intestinal microbiota of the infant that might explain the mechanism from prenatal to adult-like microbiota through delivery mode at birth, type of feeding especially at the first week and first months. ${ }^{6}$ Finally, this article aims to find an appropriate solution or intervention to promote optimal growth and development of the C-section-born infants and children in the perspective of nutrition and gut microbiota balance.

\section{Nutritional intervention to support growth and development of children born by $\mathbf{C}$-section}

It is widely agreed that human breastmilk is the gold standard as the first and main food for infants since born to be exclusively provided during the first six months and being continued up to 24 months of age. Breast milk provides complete nutrients to support child growth and development, regardless of difference in the route of delivery. Besides having all essential nutrients, breastmilk also consists of immune-components, hormones, HMOs (human milk oligo-saccharides, and microbiota. The HMOs as prebiotic and microbiota as probiotic, or synbiotic in breastmilk are important on their function to influence on the infant gut microbiota for its longterm health benefits, e.g. lung health. Figure 1 shows the hypothetical pathway on the association of HMOs as prebiotics and microbiota as probiotic to affect infants' gut microbiota and lung health. ${ }^{7}$

However, not all infants are being fortunate for having the breast milk, but instead will have its substitute, i.e. milk formula. To be updated, the milk formula is continued to be designed to mimic the breast milk, i.e. by 1) decreasing its protein content without changing plasma amino acid profile in preventing obesity; 2) supplemented with cow's milk lactoferrin; 3) having long-chain PUFA (omega-3 and pmega-6) to promote insulin sensitivity, prevention of obesity and dyslipidemia; 4) addition of milk fat globule membrane (MFGM) from cow's milk to add on to the plant oil; 5) supplementation of prebiotic (FOS and GOS) to have a bifidogenic effect; and/or 6) supplemented with probiotic in which there is no report on its harmful effects. ${ }^{8}$

The use of pre-, pro- and synbiotic in infant formula is done through research and innovation by using technological advances along with the development of knowledge about the component of breast milk. However, the evidence about its clinical efficacy is limited to recommend as a routine use in infant formula. Therefore, further research is needed to be able to establish their benefits for health. ${ }^{9}$ This recommendation is also considered to the inconsistency evidence, i.e. from 1) the negative finding of a prospective, randomized, double-blind controlled study among full term infants with diagnosed cow's mild allergy (CMA) who received synbiotic supplemented amino acid-based formula (AAF) that showed similar growth to the AAF without synbiotics,${ }^{10}$ to the positive finding from a randomized, double-blind, multicenter study on the effect of synbiotic on the microbiota of caesarean delivery infants in which supplementation with short-chain galacto-oligosaccharides/long-chain fructo-oligosaccharides and Bifidobacterium 
breve $\mathrm{M}-16 \mathrm{~V}$ compensates the delay Bifidobacterium colonization in C-section-delivered infants and modulates the production of acetate and the acidification of the gut. Physiologically, it reveals as indicator of gut health, emulate those observed in vaginally born infants. ${ }^{11}$

\section{Superiority of synbiotic compare to prebiotic or probiotic intervention alone on $\mathrm{C}$-section born children}

There are things to consider regarding to the needs to provide synbiotic intervention. Several studies showed the effects of antibiotic treatment in the prenatal period and early post-natal life in the gut microbiota, as well as on the risk of wheeze and asthma. While it also evidence that there are maternal factors influencing the composition of breast milk microbiota, and among others is Csection. All of those will induce dysbiosis condition and increase the risk of allergy. ${ }^{12}$

The dysbiosis in children born by $\mathrm{C}$-section is influenced by several factors, i.e. extremes of maternal body mass index (BMI), preterm birth, extremes of infants' size, infection, and gestational diabetes. The impact of microbial dysbiosis caused by $\mathrm{C}$-section delivery is linked with an increased risk of inflammatory bowel disease (IBD) and a wide range of autoimmune, allergic and metabolic condition, as shown in Figure 2. ${ }^{13}$

What are the potential for pre-, pro- or synbiotics in the management of infants at risk of dysbiosis? The use of prebiotic is due to its potential effect in selectively utilized by host microorganisms conferring a health benefit. While probiotic as live organisms has it potential when administered in adequate amount confer a health benefit on the host. Furthermore, synbiotic as a mixture of pre- and probiotics potentially affects the host by improving the survival and implantation of live microbial dietary supplements in the gastrointestinal tract, improving the health of the host. ${ }^{14}$ It has been recommended that during pregnancy, a probiotic treatment may represent an effective strategy to promote a healthy microbial composition in $\mathrm{C}$ section born infants, while also to give prebiotic supplementation in infants exposed to early-life microbial perturbation, such as a C-section born infant. Furthermore, the combination of pre- and probiotics or synbiotics is thought to have synergistic beneficial effects on the immune and metabolic systems. ${ }^{15}$ Thus, synbiotic should have a greater effect than the prebiotic or probiotic alone as shown in Figure 3 on the mechanism of action and their effects. ${ }^{16}$

Regarding to the mechanism of action, then while providing the synbiotic intervention to the $\mathrm{C}$ section born infants and children, we should consider to give nutritional modulation of maternal microbiota that might influence the development of the infant gastrointestinal tract. Using a hypothetical model, we can learn how maternal microbiota and microbial products could be transferred from mother to the fetal and neonatal gastrointestinal tract. It shows that dendritic cells can cross the paracellular space of the intestinal epithelium to take up bacteria directly from the intestine lumen. Following to that action then circulation of lymphocytes within the mucosal associated lymphoid tissue allows the maternal gastrointestinal tract microbiota to reach distant mucosal surfaces, including those found in the genitourinary and respiratory tracts, lactating mammary gland, salivary and lachrymal glands, as shown in Figure 4. ${ }^{17}$

This explanation inspires us to manipulate the maternal gastrointestinal microbiota composition through the use of pro-, pre or synbiotic and its subsequent impacts for the health of the newborn. To confirm the hypothesis, an Indonesian study can show the effect of probiotic provided to pregnant women since the $3^{\text {rd }}$ trimester can be found in the colostrum and the 3 -month breast milk. ${ }^{18}$

Finally, regarding to the composition of symbiotic, although we have already been informed on the properties of several microorganism considered as probiotics (such as Lactobacillus and Bifidobacterium), and prebiotics (such as oligosaccharides, i.e. FOS and GOS), however we still need further information on its combination as a synbiotic. So far, the most used and already marketed synbiotics are mixtures of oligosaccharides FOS and GOS with probiotics bacterial strain L. plantarum, L. paracasei, $L$. rhamnosus, B. bifidum or B. lactis that have been selected for the functional activities in the context of a specific combination formulation with prebiotics. ${ }^{19}$ Several studies that showed a synbiotic combination of $L$. casei and dextran prevented the 
cedar-pollen induced onset of nasal and ocular symptoms, and a combination of potato starch and L. rhamnosus reduced a disease score of atopic dermatitis, showing that there are specific synergistic effects of a combination of certain synbiotics. Therefore, future research should provide clinical evidence of certain combination formulation of synbiotics, especially to overcome dysbiosis resulted by the $\mathrm{C}$-section.

\section{Conclusion}

Beside breast-feeding, translocation from the mother's intestinal microbiota and synbiotic supplementation are amongst nutritional intervention to support growth and development of $\mathrm{C}$-section born infants and children. Regarding to the fact that synbiotic is thought to have synergistic beneficial effects on the immune and metabolic systems by compensated the delayed Bifidobacterium colonization in C-section born infants and modulates the production of acetate and the acidification of the gut, therefore, symbiotic for children in general and specifically for C-section infants and children may have an impact on healthy young children gut microbiota, although further research is certainly needed.

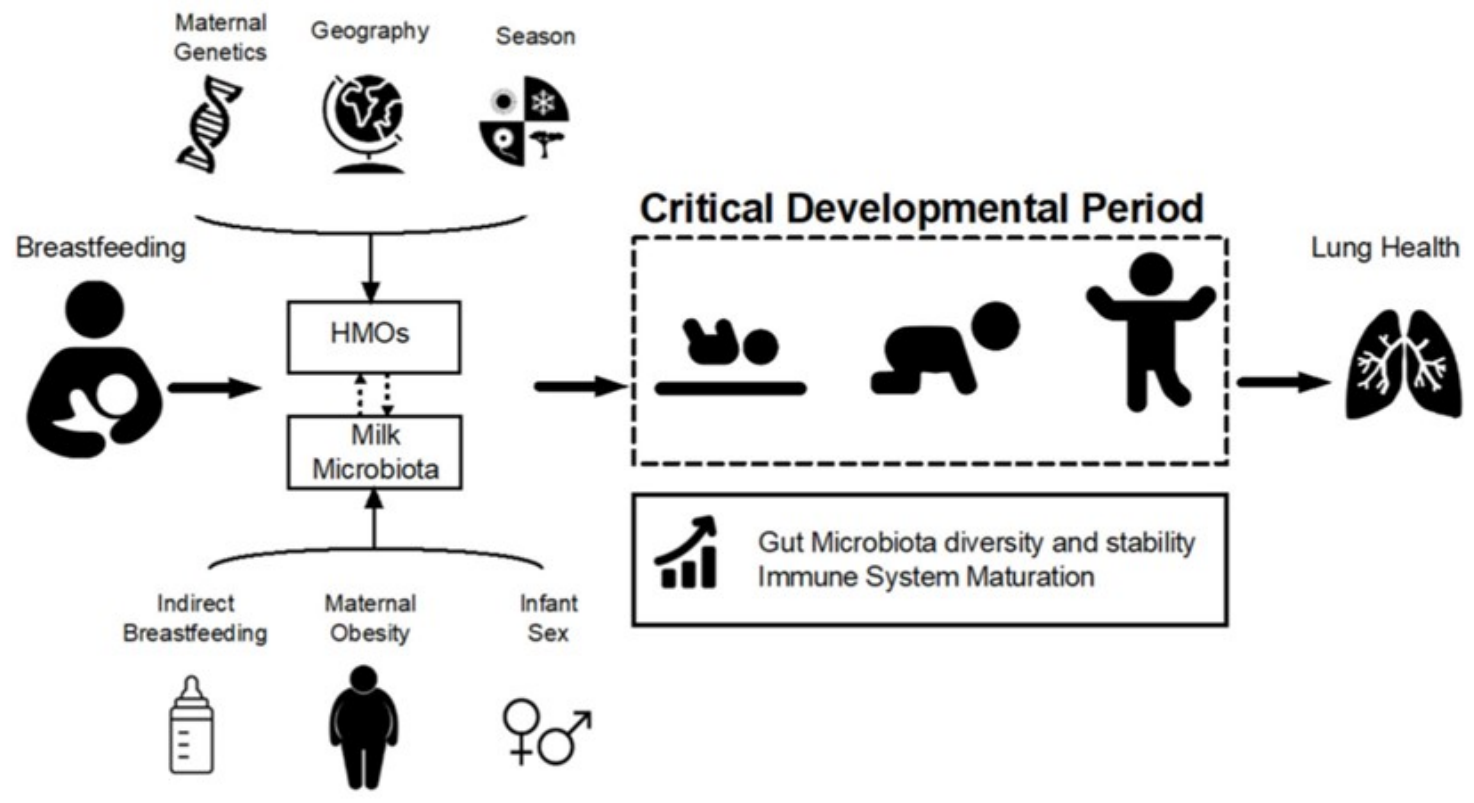

Figure 1. Hypothetical pathways of association between breastfeeding and lung health ${ }^{7}$ 


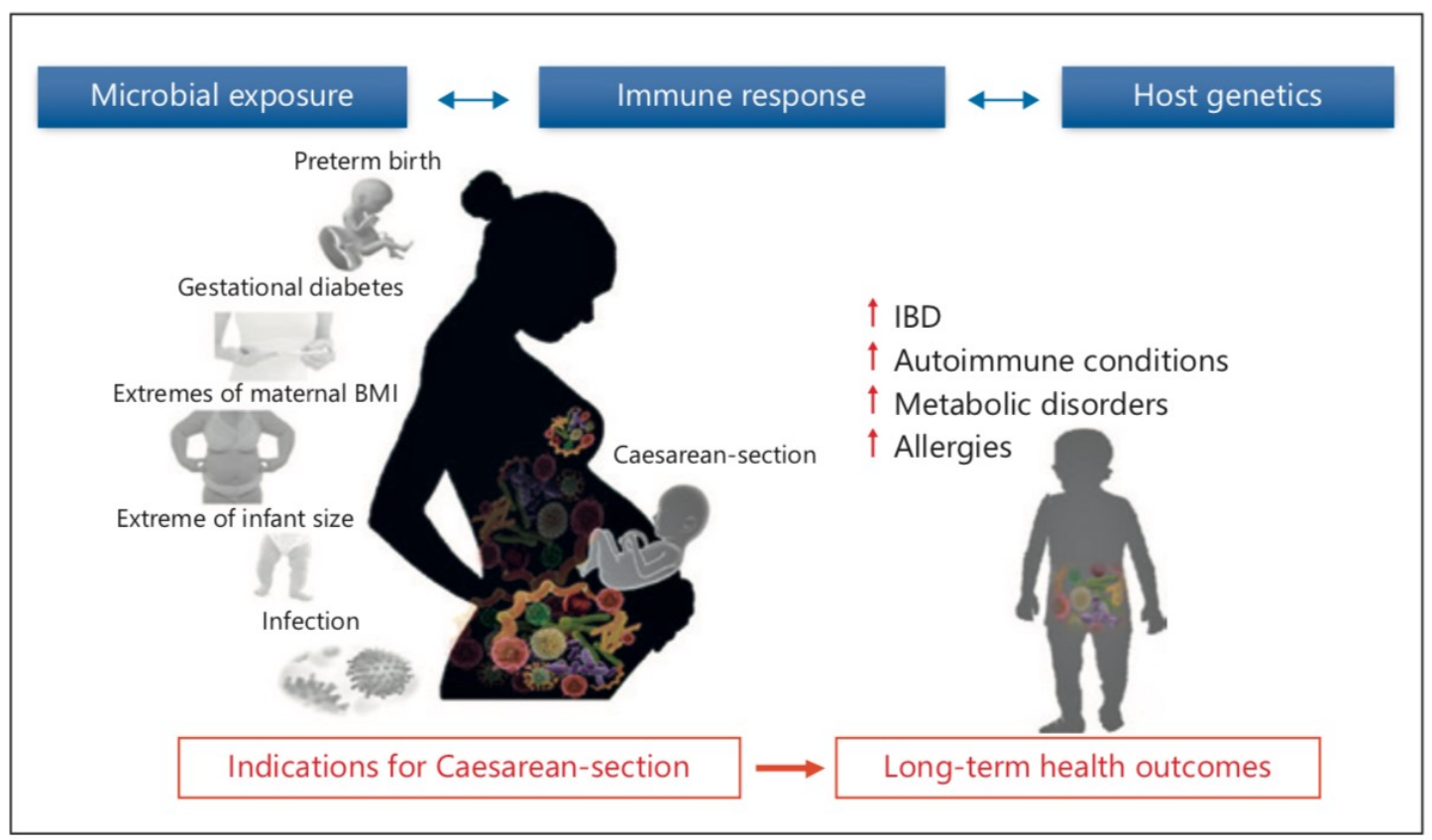

Figure 2. Contributing factors and conditions linked to C-section delivery ${ }^{13}$

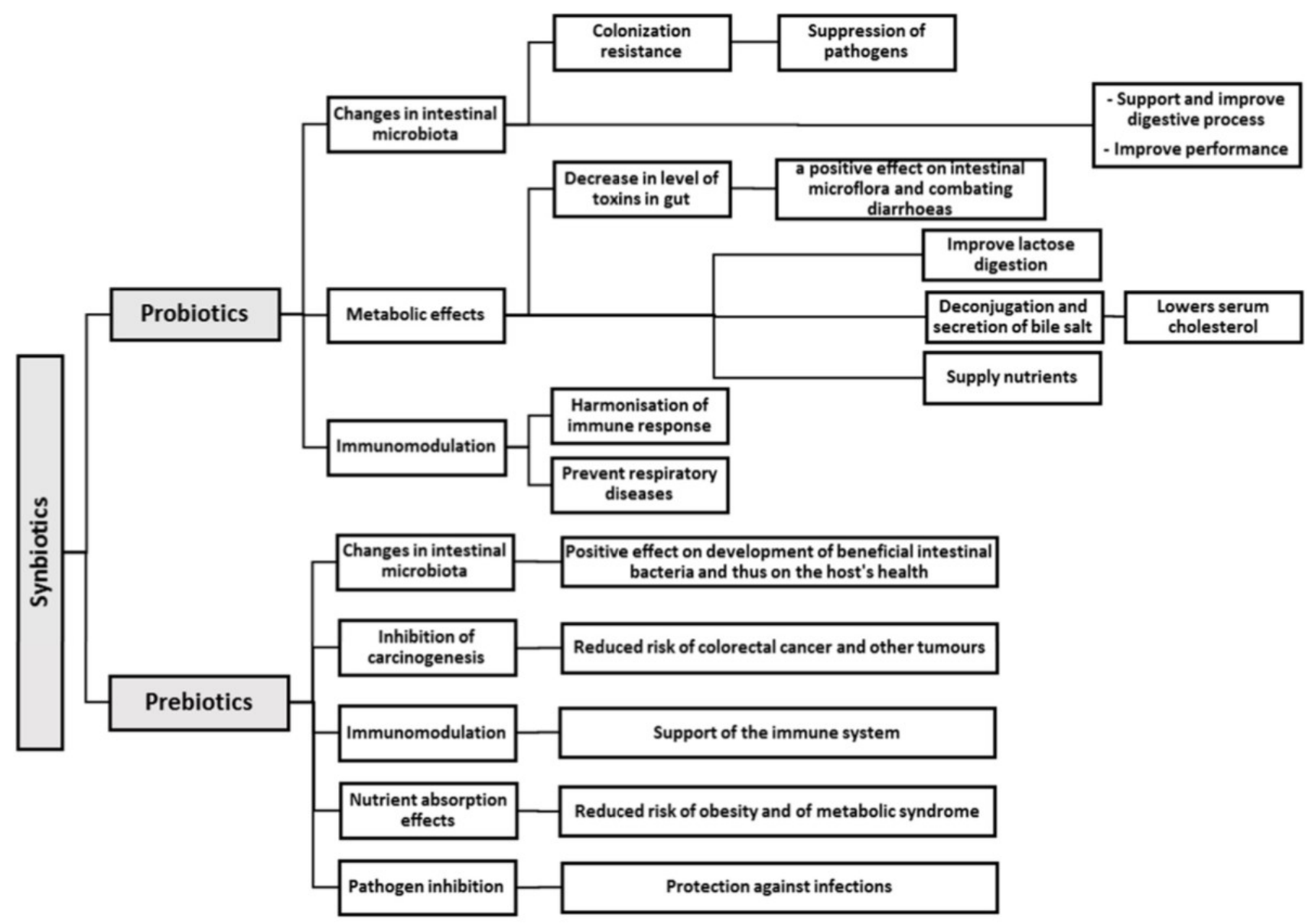

Figure 3. Mechanism of actions of synbiotics and their effects 


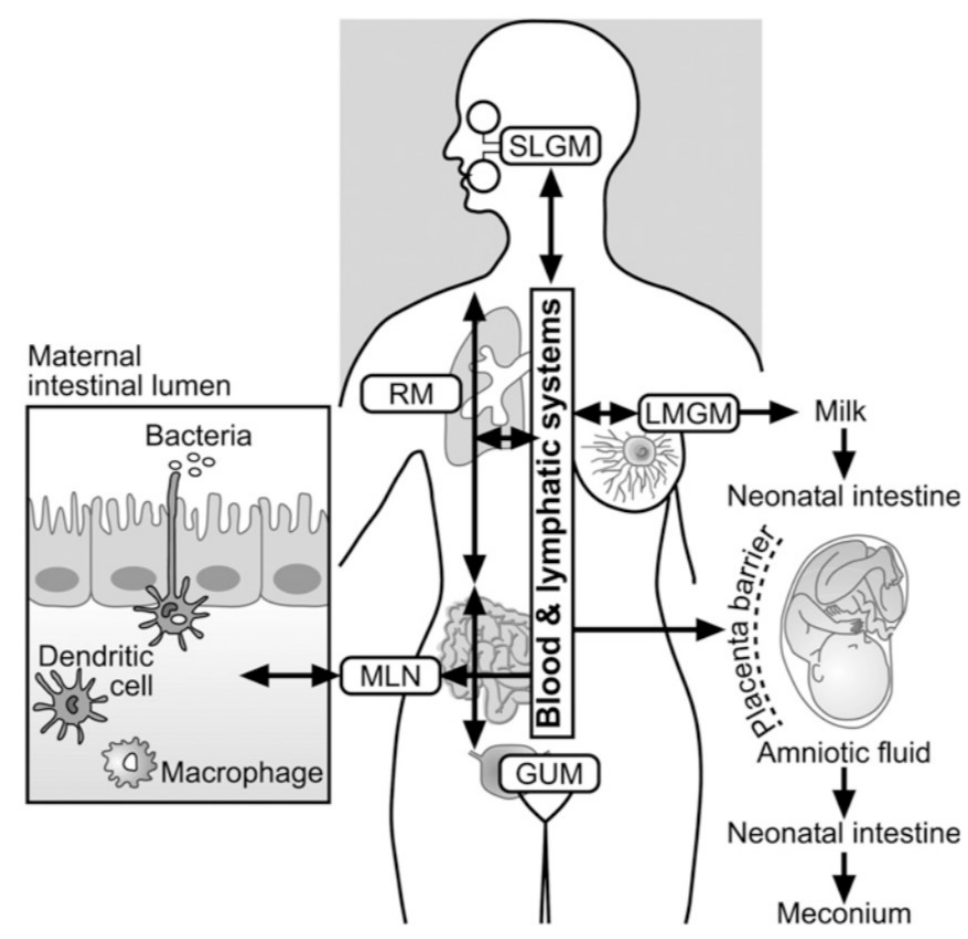

Figure 4. A hypothetical model of how maternal microbiota and microbial products may be transferred from mother to fetal's and neonatal's gastrointestinal tract

\section{Conflict of Interest}

Authors declared no conflict of interest regarding this article.

\section{Open Access}

This article is distributed under the terms of the Creative Commons Attribution 4.0 International
Licence

(http://creativecommons.org/licenses/by/4.0/), which permits unrestricted use, distribution, and reproduction in any medium, provided you give appropriate credit to the original author(s) and the source, provide a link to the Creative Commons license, and indicate if changes were made.

\section{Reference}

overweight and obesity in preschool children. $B M C$ Pregnancy Childbirth. 2016;16:338.

4 Deoni SC, Adams SH, Li X, Badger TM, Pivik RT, Glasier CM, et al. Cesarean delivery impacts infant brain development. Am J Neuroradiol. 2019;40:16977.

5 Polidano C, Zhu A, Bornstein JC. The relation between cesarean birth and child cognitive development. Sci Rep. 2017;7:11483. 
6 Metamoros S, Gras-Leguen C, Vacon FL, Postel G, de La Cochetiere M-F. Development of intestinal microbiota in infants and its impact on health. Trends Microbiol. 2013;21(4):167-73.

7 Moosavi S, Miliku K, Sepehri S, Khafipour E, Azad MB. The prebiotic and probiotic properties of human milk: Implication for infant immune development and pediatric asthma. Front Pediatr. 2018;6:197.

8 Lemaire M, Huëron-Luron IL, Blat S. Effects of infant formula composition on long-term metabolic health. $J$ Dev Orig Health Dis. 2018;9(6):573-89.

9 Federik M. Use of probiotic, prebiotic and symbiotic in infant formulas. J Nutr Hum Health. 2019;3(1):12-8.

10 Burks AW, Harthoorn LF, Van Ampting MTJ, Nijhuis MMO, Langford JE, Wopereis H, et al. Synbioticssupplemented amino acid-based formula supports adequate growth in cow's mild allergic infants. Pediatr Allergy Immunol. 2015;26:316-22.

11 Chua MC, Ben-Amor K, Lay C, Goh AEN, Chiang WC, Rao R, et al. Effect of synbiotics on the gut microbiota of caesarean delivered infants: a randomized, double-blind, multicenter study. $J$ Pediatr Gastroenterol Nutr. 2017;65:102-6.

12 Cukrowska B, Bierla JB, Zakrzewska M, Klukowski M, Maciorkowska E. The relationship between the infant gut microbiota and allergy. The role of Bifidobacterium breve and prebiotic oligosaccharides in the activation of anti-allergic mechanisms in early life. Nutrients. 2020;12:946.

13 Garcia MCS, Yee AL, Gilbert JA, Dsouza M. Dysbiosis in children born by caesarean section. Ann Nutr Metab. 2018;73(3):24-32.
14 Fox A, Bird JA, Fiocchi A, Knol J, Meyer R, ASalminen S, et al. The potential for pre-, pro-, and synbiotics in the management of infants at risk of cow's milk allergy or with cow's milk allergy: An exploration of the rationale, available evidence and remaining questions. World Allergy Organization Journal. 2019;12. 100034

15 Francavilla R, Cristofori F, Tripaldi ME, Indrio F. Intervention for dysbiois in children born by $\mathrm{C}$ section. Ann Nutr Metab 2018;73(3):33-9.

16 Markowiak P, Slizewska K. Effects of porbiotics, prebiotics, and synbiotics on human health. Nutrients 2017;9:1021.

17 Thum C, Cookson AL, Otter DE, McNabb WC, Hodgkinson AJ, Dyer $\mathrm{J}$, et al. Can nutritional modulation of maternal intestinal microbiota influence the development of the infant gastrointestinal tract. J Nutr. 2012;142:1921-8.

18 Dewanto NEF, Firmansyah A, Sungkar A, Dharmasetiawani N, Sastroasmoro S, Kresno SB, et al. Effect of Bifidobacterium animalis HNO19 supplementation among pregnant and lactating women on interleukin-8 level in breast milk and infant's gut mucosal integrity. Med $J$ Indones. 2017;26:204-11.

19 Gupta C, Prakash D, Rostagno MH, Callaway TR. Synbiotics: Promoting gastro-intestinal health. Phytochemicals of Nutraceutical Importance. 2014;61-78 